\title{
Bilateral ONJ in a patient affected by metastatic prostate adenocarcinoma.
}

\author{
Paolo Boffano ${ }^{1}$, ANNA MARIA AGNONE, Francesco Cavarra, ANDREA MELLE ${ }^{2}$, Vincenzo Rocchetti ${ }^{2}$, Tricarico \\ Gerardo $^{1}$
}

1 ASL VC

2 University of Eastern Piedmont

Funding: The author(s) received no specific funding for this work.

Potential competing interests: The author(s) declared that no potential competing interests exist.

\section{Abstract}

We present the diagnosis and management of a case of bilateral $\mathrm{ONJ}$ of the mandible in a patient that had been taking zoledronic acid treatment for 7 years following a diagnosis of metastatic prostate adenocarcinoma.

The patient was referred to our Division by his dental practitioner, that had performed the extraction of the left lower first molar six months before. In the first months after the extraction the patient did not reported any symptom and he started to use a mandibular removable prosthesis.

About 5 months following the extraction, the patient started to complain of pain in correspondence of the left body region. He had performed a panoramic radiograph and a CT scan showing a possible initial stage of mandibular ONJ bilaterally, with a predominance in the left mandibular body in the post-extraction socket. Therefore, a curettage of the left mandibular body was decided. Postoperative course was uneventful. Twelve months later, the patient complained of pain in the right mandibular region and in correspondence of the residual right lower third molar. A panoramic radiograph confirmed the extension of mandibular right ONJ. A curettage of the right mandibular body, together with the removal of the right lower third molar, was performed, with a following uneventful postoperative course.

Two years later, the patient complained of pain in the left mandibular region in correspondence of the residual left lower second premolar. A panoramic radiograph confirmed the extension of mandibular left ONJ with involvement of the root of the aforementioned premolar. A curettage of the right mandibular body, together with the removal of the left lower second premolar, was performed, with a following uneventful postoperative course. Three years later, a complete healing was obtained.

Background: Bisphosphonates are used in cancer patients to decrease and delay complications associated with metastasis, thanks to their binding activity to hydroxyapatite and their capacity to inhibit bone turnover by interfering with osteoclastic activity. Therefore, bisphosphonates are used to prevent and reduce hypercalcemia, pain, and pathologic fractures. Nevertheless, bisphosphonates may be associated with a considerable risk for the development of osteonecrosis of the jaw (ONJ). 
Different etiological factors have been reported to determine ONJ, such as dental extractions, the placement of dental implants, and the use of dental prosthesis. The aim of this article was to present and discuss the diagnosis, management, and follow up of a patient treated with zoledronic acid because of a metastatic prostate adenocarcinoma, that developed bilateral mandibular ONJ.

Materials and Methods: A patient affected with metastatic prostate adenocarcinoma (Gleason score 8) and treated with zoledronate was referred to Our Division by his dental practitioner. He was given zoledronate by the intramuscular route since 5 years.

His dentist had performed the extraction of the left lower first molar six months before. In the first months after the extraction the patient did not reported any symptom and he started to use a mandibular removable prosthesis.

About 5 months following the extraction, the patient started to complain of pain in correspondence of the left body region. He had performed a panoramic radiograph and a CT scan showing a possible initial stage of mandibular ONJ bilaterally, with a predominance in the left mandibular body in the post-extraction socket.

Therefore, a curettage of the left mandibular body was decided. Postoperative course was uneventful.

Twelve months later, the patient complained of pain in the right mandibular region and in correspondence of the residual right lower third molar. A panoramic radiograph confirmed the extension of mandibular right ONJ. A curettage of the right mandibular body, together with the removal of the right lower third molar, was performed, with a following uneventful postoperative course.

Two years later, the patient complained of pain in the left mandibular region in correspondence of the residual left lower second premolar. A panoramic radiograph confirmed the extension of mandibular left ONJ with involvement of the root of the aforementioned premolar. A curettage of the right mandibular body, together with the removal of the left lower second premolar, was performed, with a following uneventful postoperative course.

Three years later, a complete healing was obtained

Conclusions: Our case showed the importance of an appropriate assessment of risk factors, that is not only a dentoalveolar procedure but also the use of a mobile prosthesis, that in the presented patient determined osteonecrosis in the right mandibular body. In conclusion, a great attention should be made to all risk factors for ONJ, as our case shows. Dental practitioners should take care of both dentoalveolar surgery and dentures in patients that assume bisphosphonates 